www.refaad.com
المجلة الدولية للدراسات القانونية والفقهية المقارنة

International Journal of Legal and Comparative Jurisprudence Studies (LCJS)

Journal Homepage: https://www.refaad.com/views/LCJS/Home.aspx

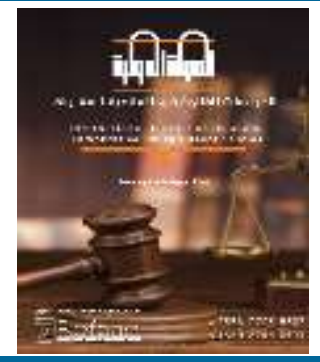

\title{
مدى كفاية التشريعات الإلكترونية للحدل من التنمر الإلكتروني- دراسة في التشريع الأردني
}

\author{
صخر أحمد الخصياونة \\ تشريعات إعلامية- معهد الإعلام الأردني- عمان- الأردن \\ sakherkhasawneh1234@gmail.com
}

DOI: https://doi.org/DOI:10.31559/LCJS2020.1.2.1

الملتخص:

تستعرض الدراسة التشريعات الإلكترونية التي وضعها المشرع الأردني والتي تعمل على الحد من التنمر الإلكتروني، حيث تناولت مفهوم التنمر بشكل عام والتنمر الإلكتروني على وجاء الخصوص، ومدى كفاية التشريعات الإلكترونية للحد من التنمر الإلكتروني، والتعرف على مصطيح التهني التنمر

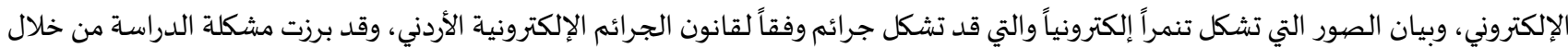
الإجابة عن السؤال الرئيس الذي نصها: "ما مدى كفاية التشريعات القانونية الأردنية للحد من التنمر الإلكتروني.

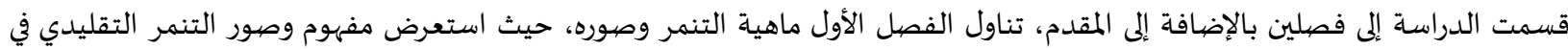
المبحث الأول، ومفهوم وصور التنمر الإلكتروني في المبحث الثاني، أما الفصل الثاني فقد استعرض التكييف القانوني لأنواع واشكال التنمر الإلكتروني،

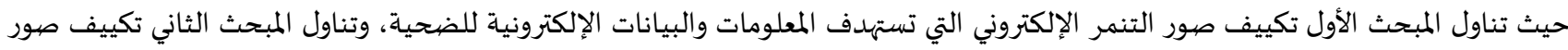
التنمر الإلكتروني التي تستهدف المعلومات والبيانات الإلكترونية للضحية الضية.

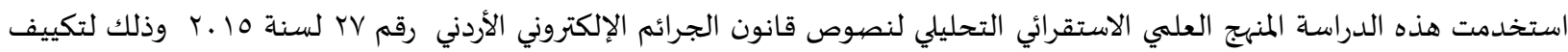
الصهور والأشكال التي تندرج تحت مسمى التنمر الإلكتروني والتي قد تشكل جريمة يعاقب عليها التهائ القانون. تبين من خلال هذه الدراسة أن معظم صور وأشكال التنمر تندرج تحت نصوص يعاقب عليها قانون الجرائم الإلكترونية، وأن قانون الجرائم

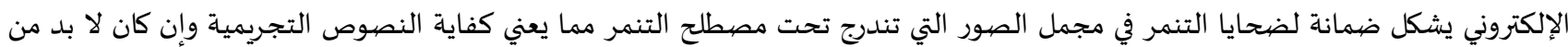
تغليظ العقوبات لبعض الصور لحماية القصر من التنمر الجنسي. الكلمات المفتاحية: التنمر الإلكتروني؛ التشريعات الإلكترونية؛ التشريع الأردني. (ㄷ) (1)

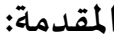

تعتبر مواقع التواصل الاجتماعي فضاءات ومساحات لممارسة حق الرأي والتعبير المكفولة في المواثيق الدولية والدساتير الوطنية، وهي واقع

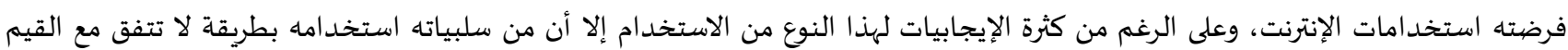

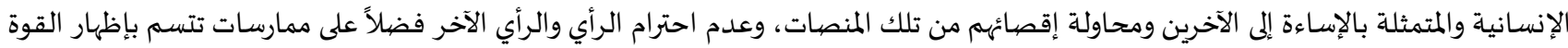

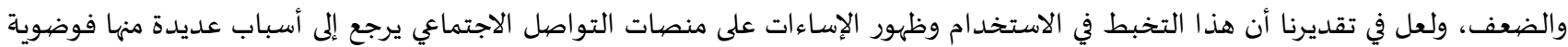

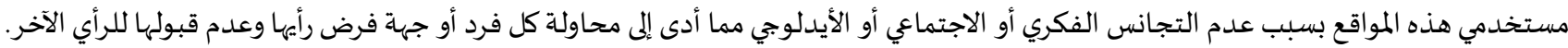

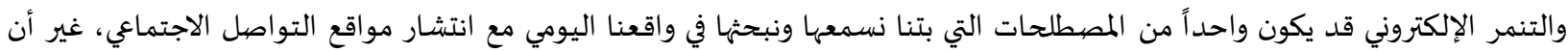
ظاهرة التنمر ليست بالجديدة إلا أن مكان التنمر والشخص المتنمر وضحية التنمر لحق بهم تغيرات، فقد تطورت هذه الظاهرة من الشكل التقليدي

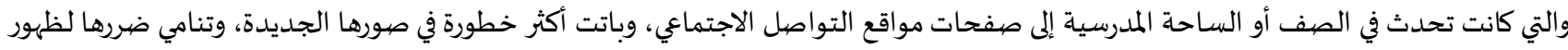

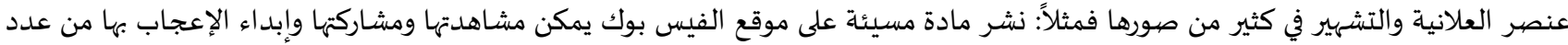


كبير من أصدقاء الصفحة(1)، وهو ما يشكل تنمراً بحق الشخص المتنمر عليه أو الضحية مما يوجب إيجاد صيغة قانونية لحفظ حقوق هؤلاء الأشخاص.

بشكل عام لا توجد إحصائيات دقيقة أو أرقام تفصيلية فيما يتعلق بالتنمر الإلكتروني في الأردن لوقوع هذه الجريمة ضمن مجموعة الجرائم

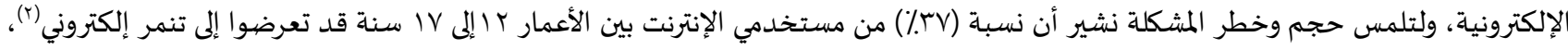

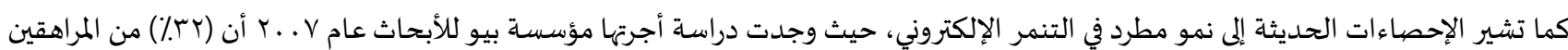
كانوا ضحايا لنوع من أنواع التنمر الإلكتروني.

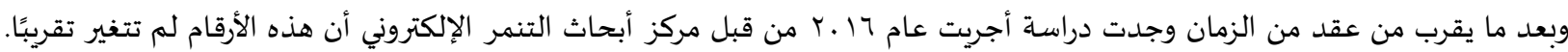

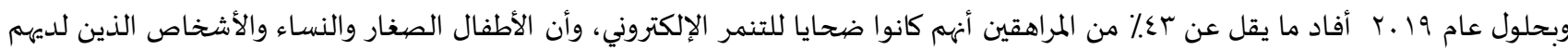

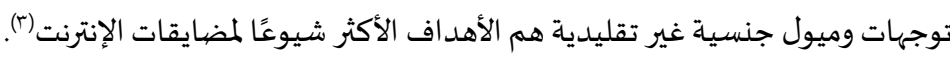

أهمية الدراسـة: تكمن أهمية هذه الدراسـة في قلة الدراسات السابقة وندرتها حول الموضوع التنمر الإلكتروني من الناحية القانونية، إذ يرى الباحث وفي حدود

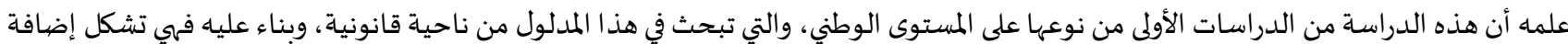

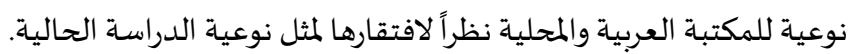
كما تكمن أهمية هذه الدراسـة في التعرف إلى مدى كفاية التشريعات القانونية الأردنية للحد من التنمر الإلكتروني، وبيان مدلول التنمر

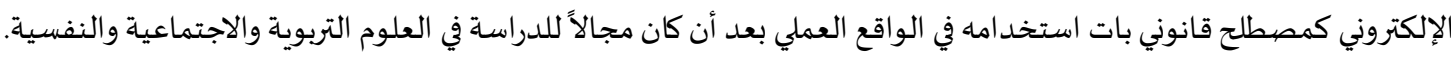

أهداف الدراسة: تهدف هذه الدراسـة إلى بيان مدى كفاية التشريعات القانونية الأردنية للحد من هذه ظاهرة التنمر الإلكتروني، وذلك من خلال: ا ـ. التعرف إلى مدلول التنمر الإلكتروني وبيان أشكاله وأنواعاه. r. البحث بالتكييف القانوني لصور وأنماط التنمر الإلكتروني وفقاً لقانون الجرائم الإلكتروني.

مشكلة الدراسة وأسئلتها: بات استخدام مصطلح التنمر الإلكتروني شائعاً لدى الصحافة وعلى لسان العامة ويتم استخدامه بشكل لافت على مواقع التواصل الاجتماعي،

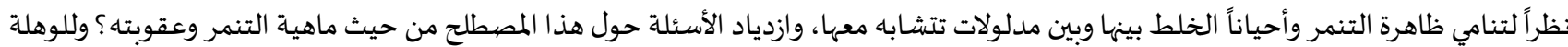

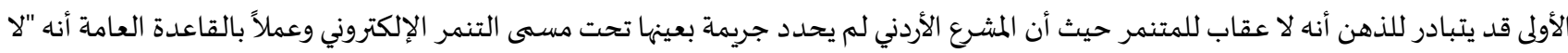

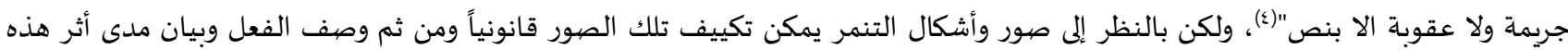
النصوص على الحد من هذه الظاهر.

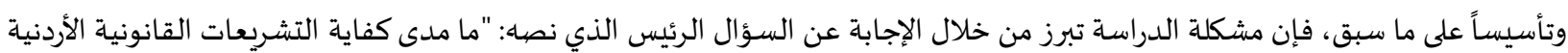

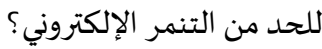

ويتفرع من هذا السؤال الرئيس، الأسئلة الفرعية الآتية:

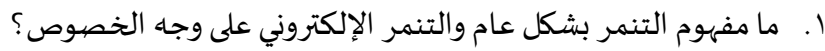

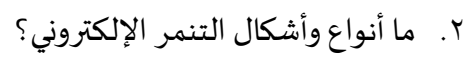

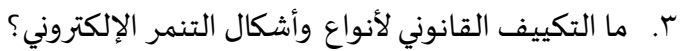

منهج الدراسة:

اتبعت الدراسة المنهج العلمي الاستقرائي التحليلي لنصوص قانون الجرائم الإلكتروني الأردني رقم VT لسنة 0 ـ ـ وذلك لتكييف الصيور والأنماط

التي قد تشكل صوراً للتنمر الإلكتروني.

${ }^{1}$ Rosa Mcphee , 2014 Sticks stones may break my bones , but cyberbullying is illegal - is cyberbullying a crime m and should be .p.4

${ }^{2}$ https://www.dosomething.org/us/facts/11-facts-about-cyber-

bullying?fbclid=IwAR3lE9b]GsGd170ltMjYLj5GOfv]3HgYBvKgPRZosTyzzWSXAyOpMoGiZE, Date of entry (22/06/2020)

${ }^{3}$ Cyberbullying Statistics, Facts, and Trends in 2020, https://firstsiteguide.com/cyberbullying-stats/, Date of entry (10/07/2020)

كُ ألمادة (r) من قانون العقوبات الأردني. 
الفصل التمهييدي:

التنمر ظاهرة قديمة متجددة عابرة للمجتمعات والثقافات، تمارس من قبل فئة أو شخص أكثر قوة على الأشخاص أو الفئات الأقل قوة(0)، أما التنمر الإلكتروني فظهر بظهور مواقع التواصل الاجتماعي واستخدام الوسائل الإلكترونية، ولبيان المقصود بالتنمر سنقسم هذا الفصل إلى مبحثين:

المبحث الأول: مفهوم التنمر التقليدي وصوره

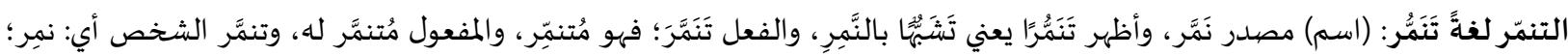

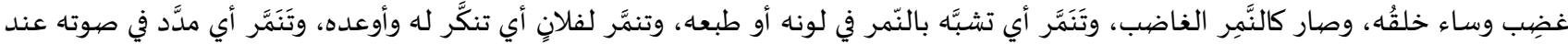

و التنمر شكل من أشكال الإسـاءة والإيذاء موجّه من قبل فرد أو مجموعة نحو فرد أو مجموعة تكون أضعف جسديًا في الغالب، والتنمر هو من

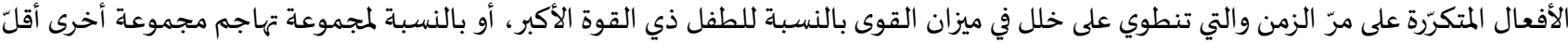

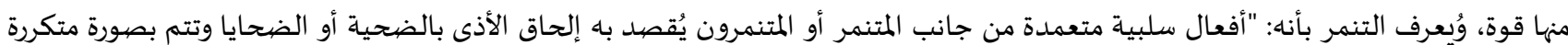
وطوال الوقت، ويجد الضحية صعوبة في الدفاع عن نفسه، ويتضمن التنمر وجود خلل في ميزان القوة بين المتنمر والضحية، بالإضافة إلى تكرار

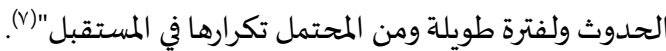

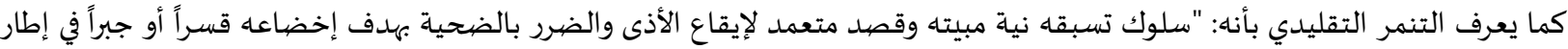
علاقة غير متكافئة ينجم عنها أضرار جسمية ونفسية وجنسية بطريقة متعمدة في مواقف تقتضي القوة والسيطرة على الضحية"(())، وهو كذلك:

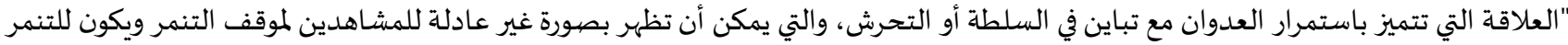

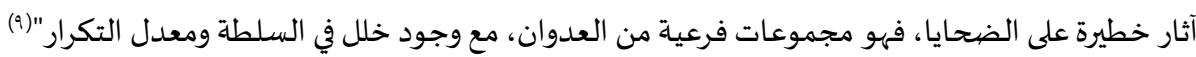

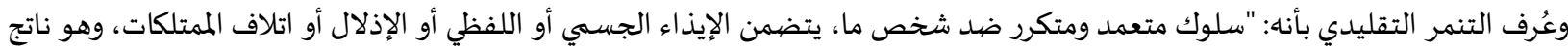

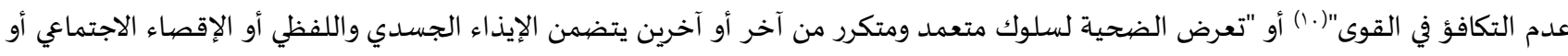
التحرش الجنسي"(11) (11). ويلاحظ من خلال ما سبق أن التنمر يقتضي القيام بأعمال عدوانية بهدف إلحاق الضرر بالضحية ويظهر من تعريفات التربويين وعلماء النفس

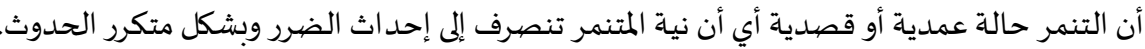

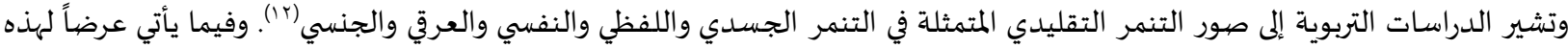

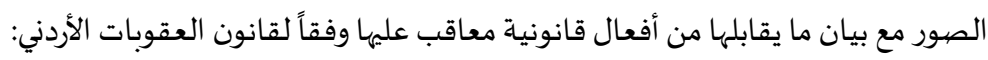

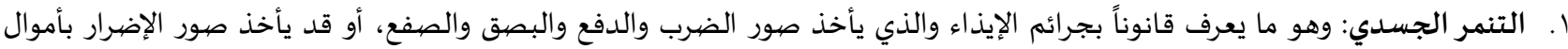
الآخرين كإتلاف الأموال الخاصة. r. التنمر اللفظي: وهو ما يشمل النعوت، والتلقيب، والإهانة، والترهيب، والتجريح، والتهديد، والتعيير، والتعييب، والذي تشكل به الأفعال السـابقة

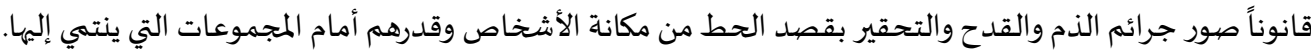

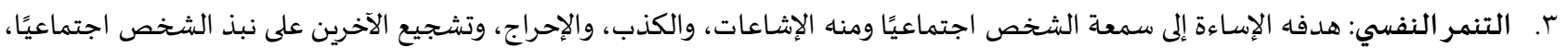

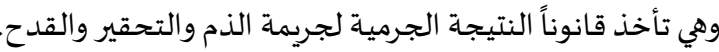

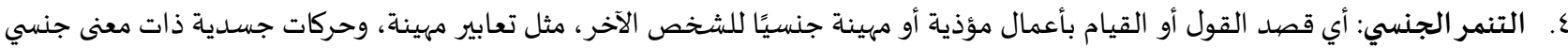

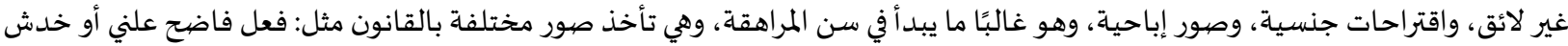

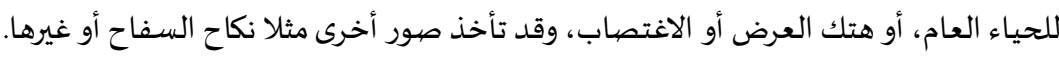

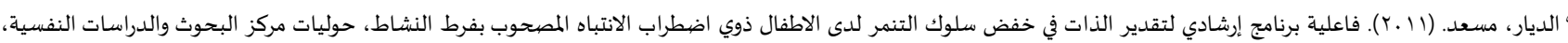
جامعة القاهرة $70-1$ ( 10

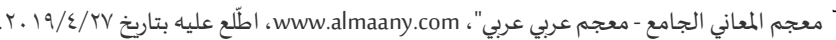
${ }^{7}$ Olweus, D. (1993). Bullying at school: what we know and what we can do. Wiley-Blackwell : oxford.

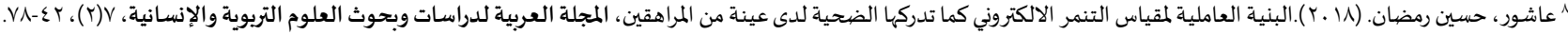

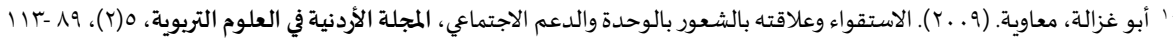

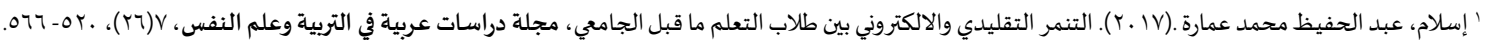
12 Beran,T \&LI .Q. (2005). Cyber-Harassment: Study of a new method for an old behavior. Journal of Educational computing Research, 32 (3) 265-277 
ه. التنمّر العرقي: وهو التنمّر على عرق أو دين أو لون أو جنس الشخص الآخر، وقد يصل هذا النوع من التنمّر إلى اشتمال كل أنواع التنمّر المذكورة

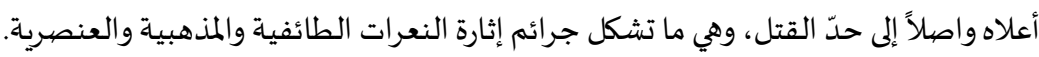

المبحث الثاني: مفهوم التنمر الإلكتروني وصهوره

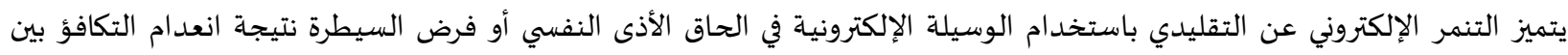
الضحية والمتنمر لذلك عُرف بأنه: "إرسال أو نشر نصوص عن أو صور ضارة عبر شبكة الإنترنت أو غيرها من الوسائل الرقمية ويتضمن المضيايقة ونشر فئري

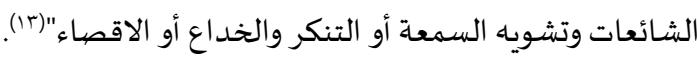
فالتنمر الإلكتروني سلوك عدواني يتم عبر الإنترنت أو وسائل الإعلام الإلكترونية أو الرقمية، والذي يقوم به فرد أو جماعة من خلال الاتصاء الإل التهال

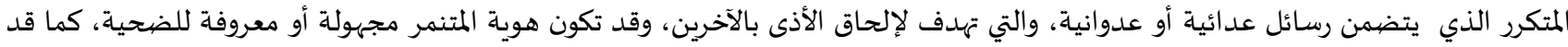

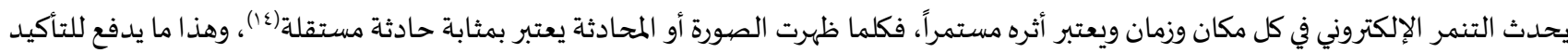

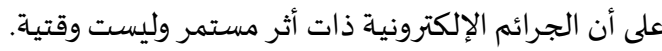

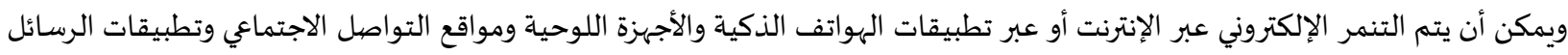

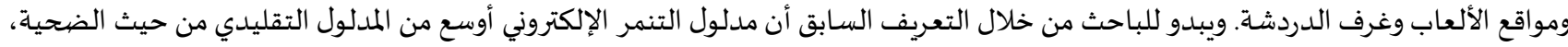

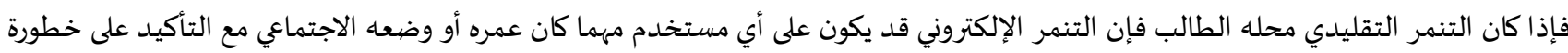
التنمر الذي يقع على الأطفال، بالإضافة إلى الأثر من حيث سعة الانتشار وظهور العلانية في تصرفات المتنمر المتسلط.

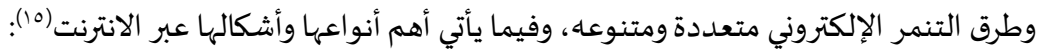

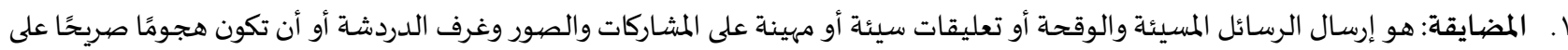
مواقع الألعاب.

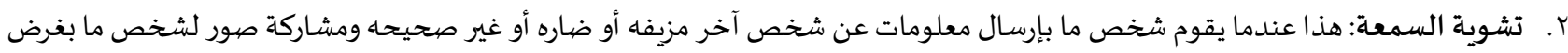

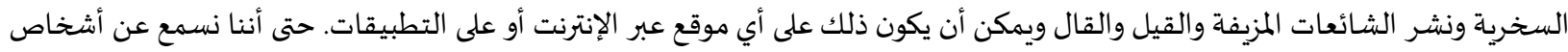

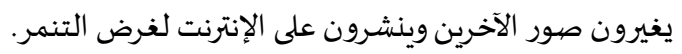

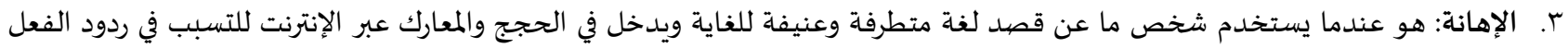

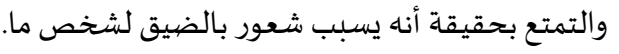

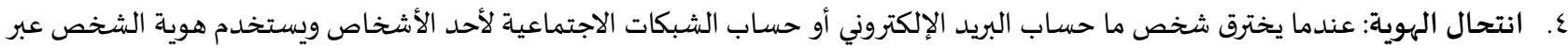

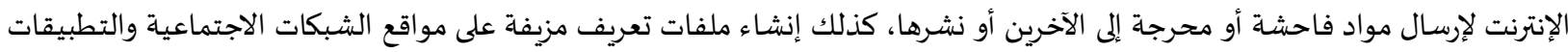
ويكون من الصعب جدًا إيقافها.

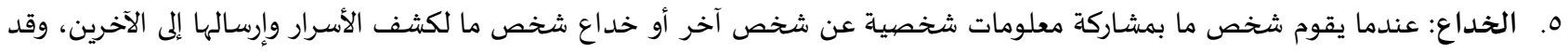

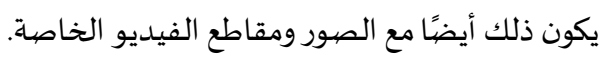

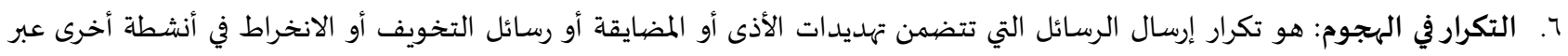
الإنترنت تجعل الشخص خائفاً على سلامته.

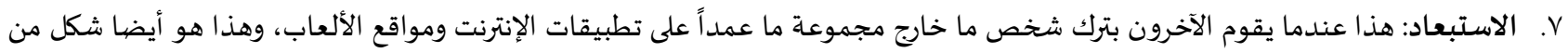
أشكال التنمر الاجتماعي وشائع جداً.

الفصل الثاني: التكييف القانوني لأنواع واشكال التنمر الإلكتروني يتضح من خلال تعرفنا على مفهوم التنمر الإلكتروني وأنماطه وأشكاله بأنه يتم عبر الوسيلة الإلكترونية أي أنه يتم من خلال مجموعة البرامج

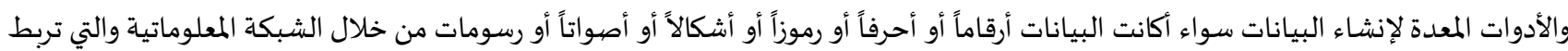

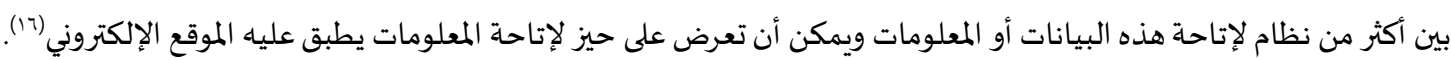

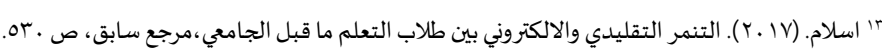
${ }^{14}$ Rosa, 2014 Sticks stones may break my bones, but cyberbullying is illegal Ibd, p5.

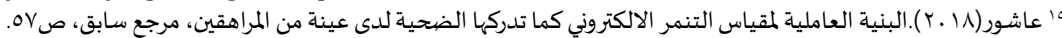

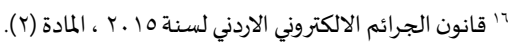




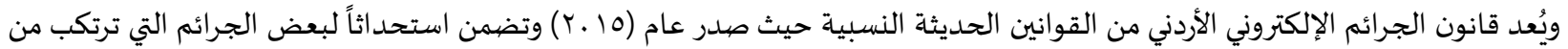

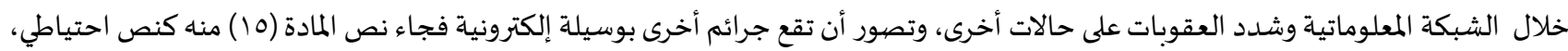

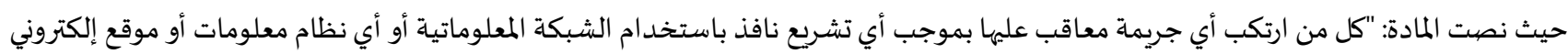

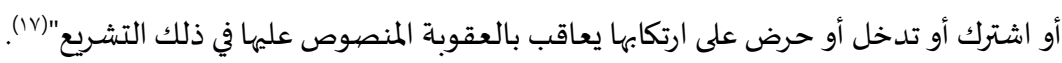

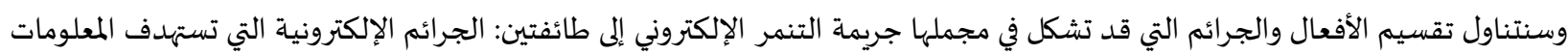
والبيانات الإلكترونية نفسها للضحية، والجرائم الإلكترونية التي تقع باستخدام الوسائل الإلكترونية على الضحية. وذلك في مطلبين:

المبحث الأول: تكييف صور التنمر الإلكتروني التي تستهدف المعلومات والبيانات الإلكترونية للضحية

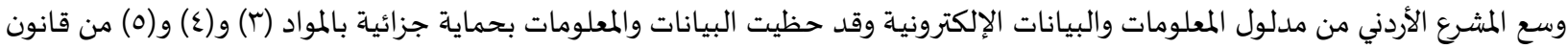

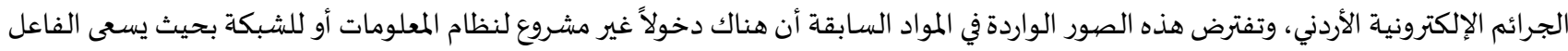

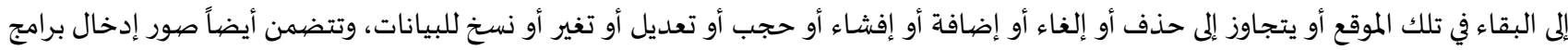

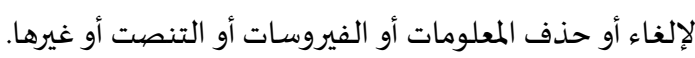

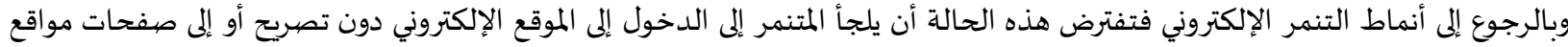

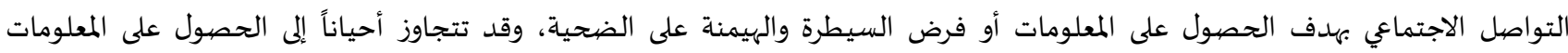

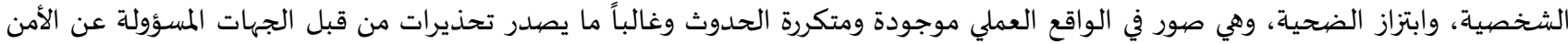

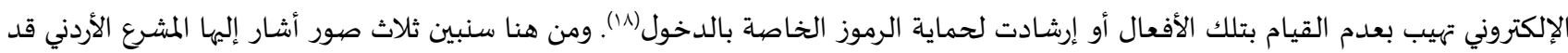
يستعملها المتنمر تشكل جرماً يعاقب عليه القانون وهي:

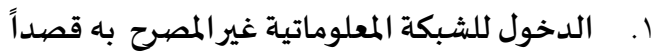

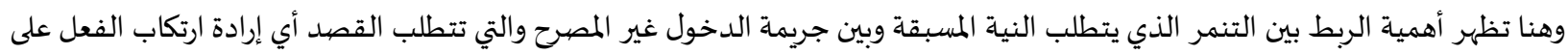

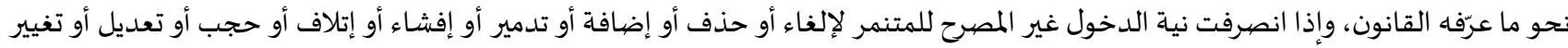

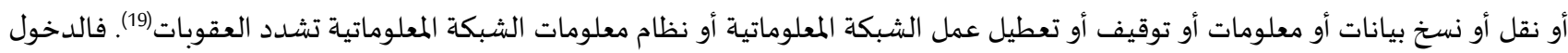
غير المصرح أو بما يسمى تهكير الحسابات وللغايات التي أوردها المشرع من قبل المتنمر كفيلة بأن تشكل أركان جريمة الدخول غير المصرح بركنيهات الماتيا المادي

وتشدد العقوبات إذا جرى انتحال صفة صاحب الموقع أو شخصية مالك الموقع الإلكتروني بحيث تصبح العقوبة الحبس مدة لا تقل عن ثلاثة

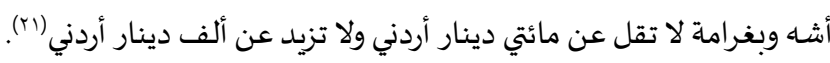

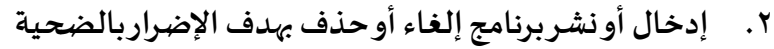

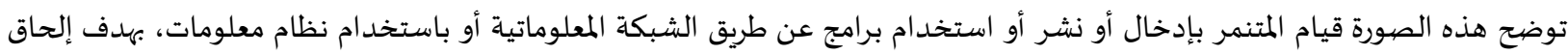

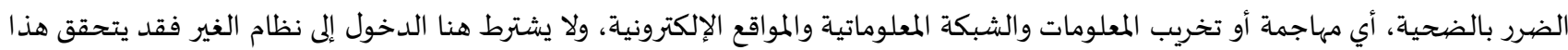

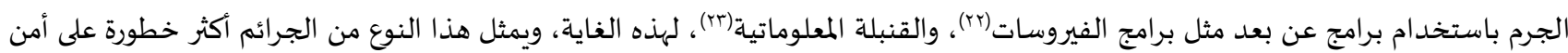

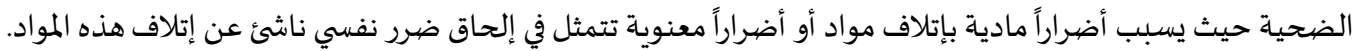

${ }^{18}$ https://royanews.tv/news/186142, Date of entry (12/07/2020)

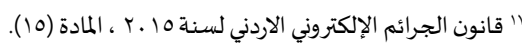
9 أنصت المادة ب من قانون الجرائم الالكتروني، على:

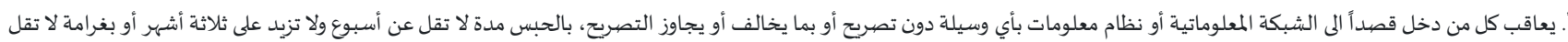

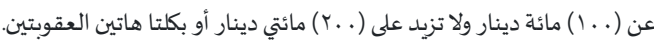

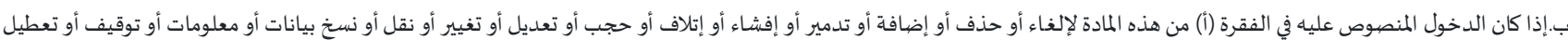

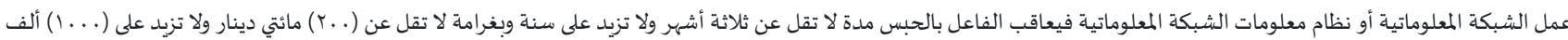

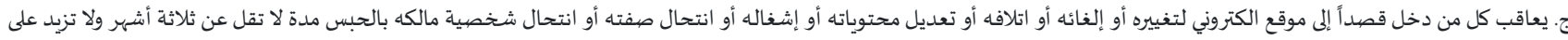

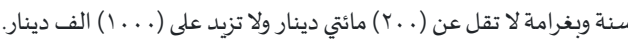

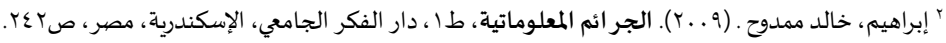

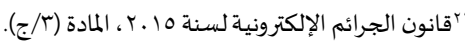

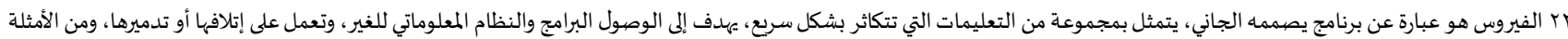

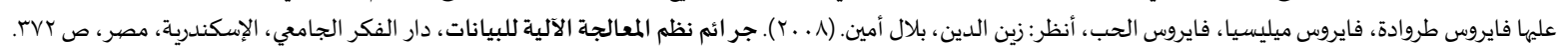




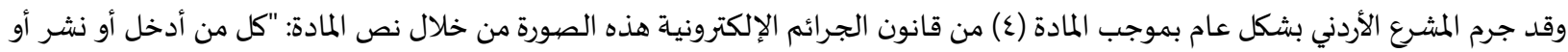

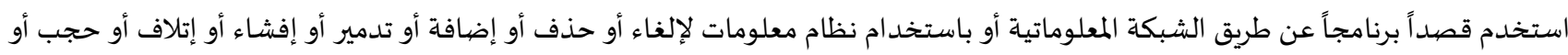

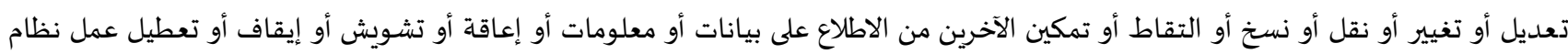

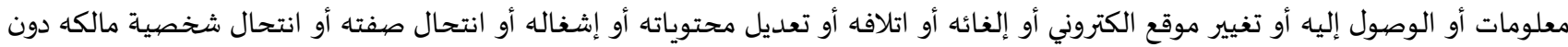

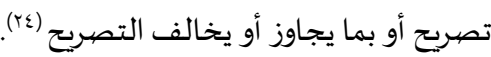
r. التقاط أو باعتراض أو بالتنصت أو أعاق أوحور أوشطب محتويات على ما هو مرسل عن طريق الشبكة المعلوماتية أو أي نظام معلومات:

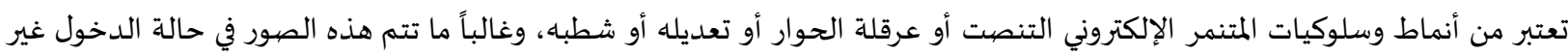

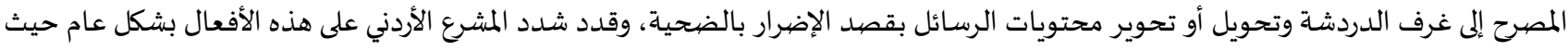

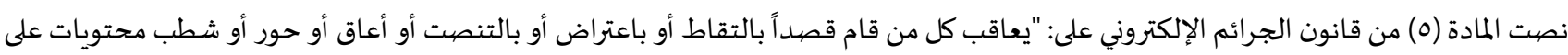

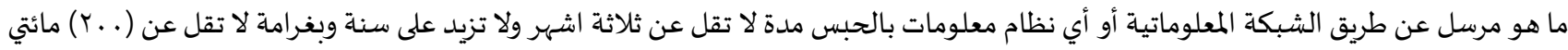

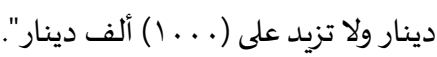

المبحث الثاني: تكييف صور التنمر الإلكتروني التي تستهدف المعلومات والبيانات الإلكترونية للضحية

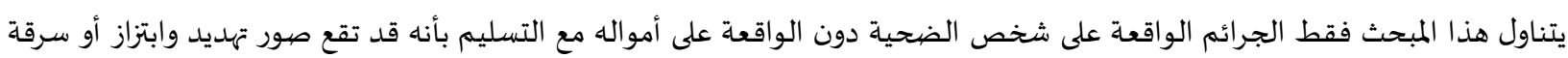

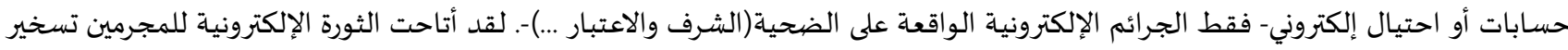

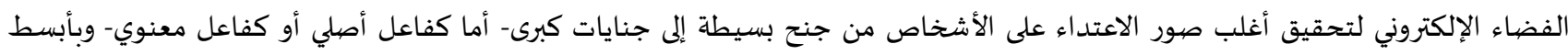

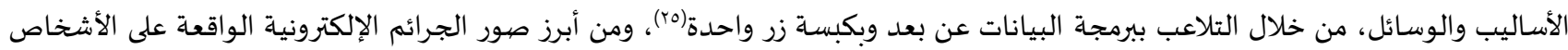

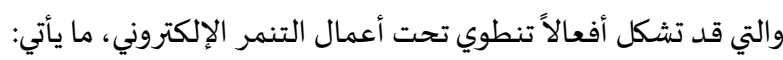

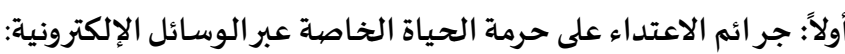

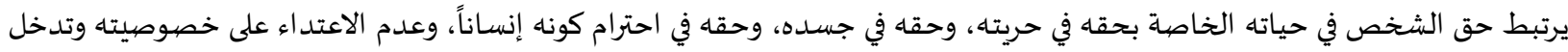

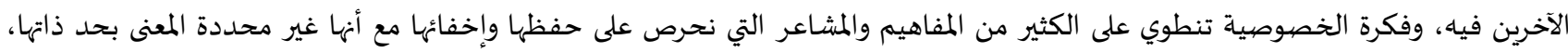

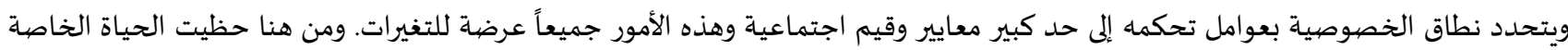

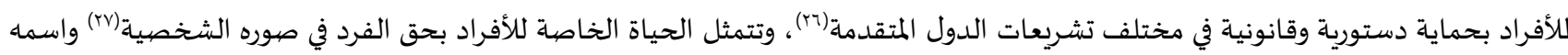

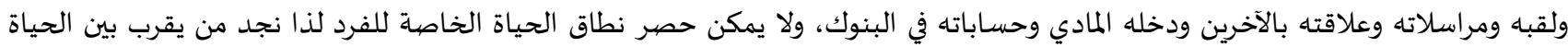

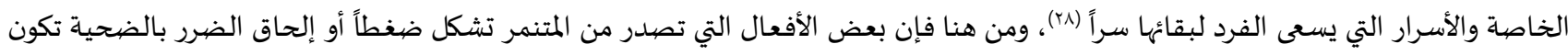

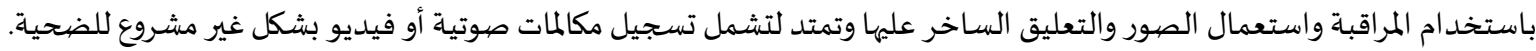

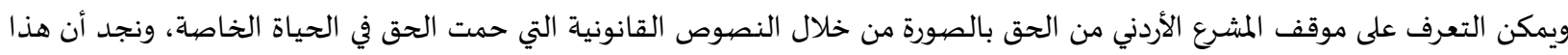

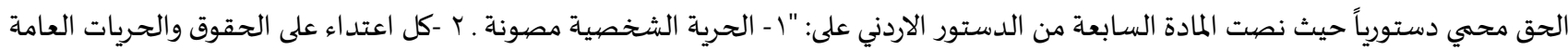

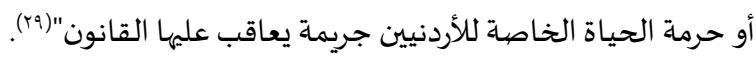

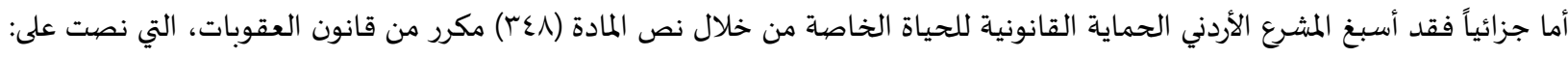

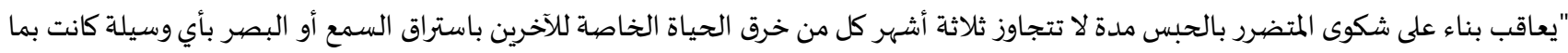

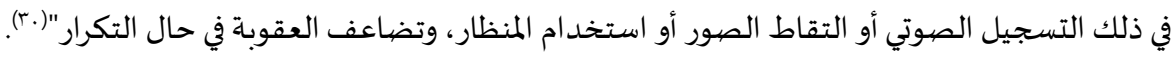

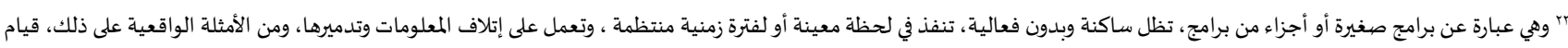

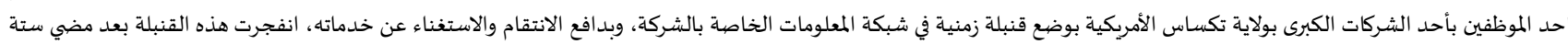

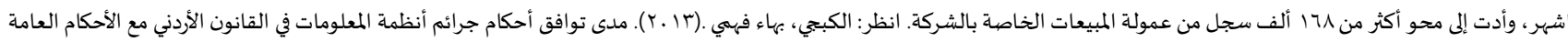

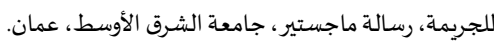

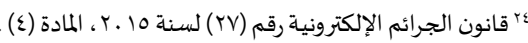

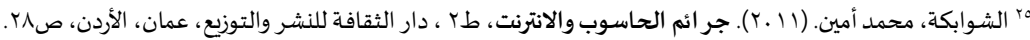

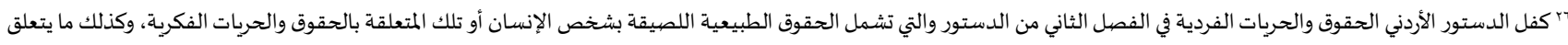

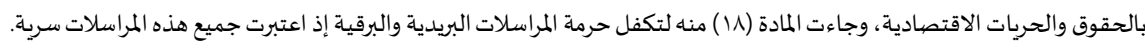

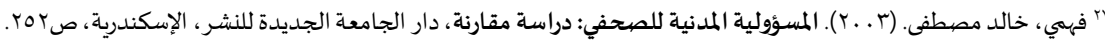

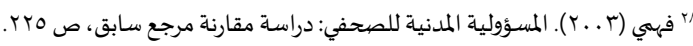

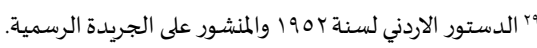

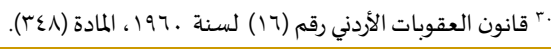


ويلاحظ هنا أن المشرع اعتبر التقاط الصور من صور خرق الحياة الخاصة. وإذا ما ارتكب الفعل بالوسائل الإلكترونية فإن المتنمر يعاقب بدلالة المالة

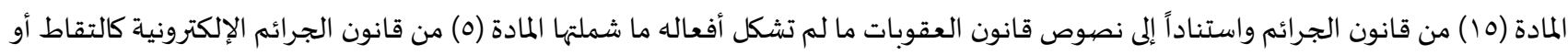

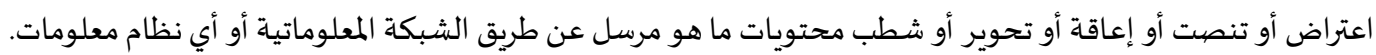

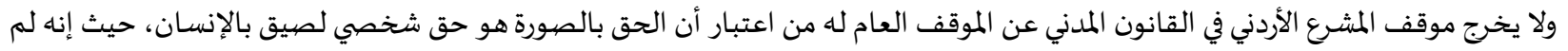

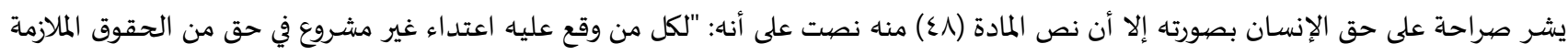

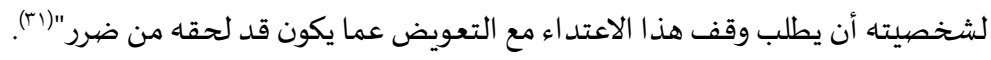

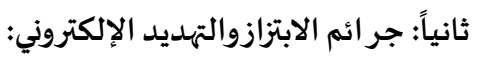

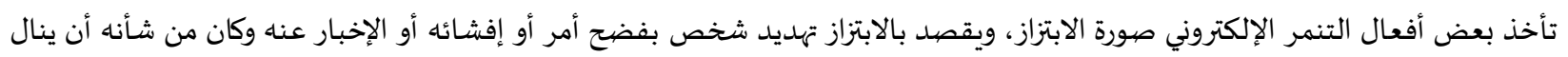

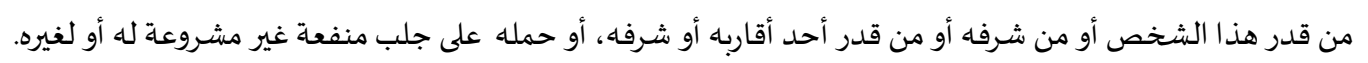

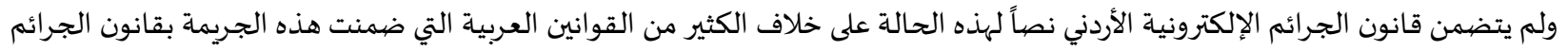

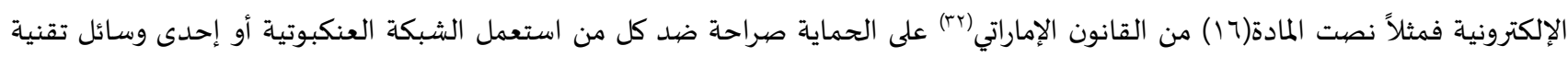

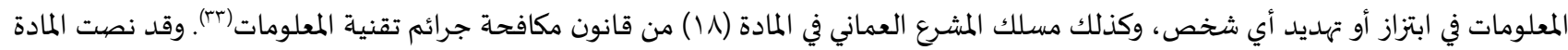

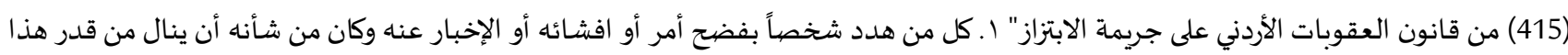

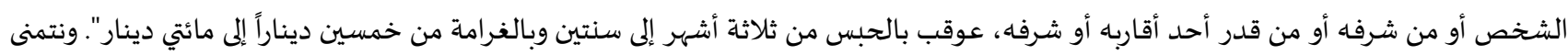

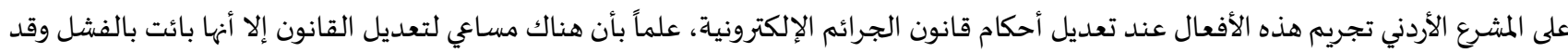

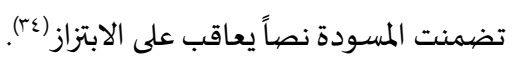

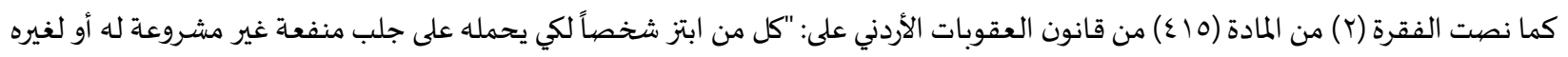

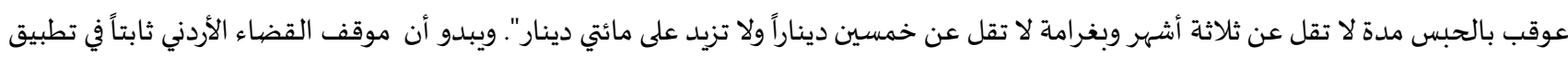

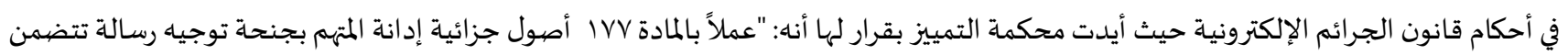

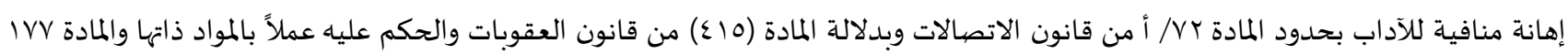

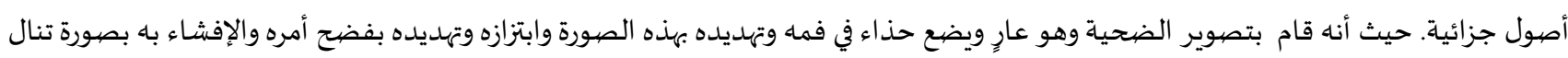

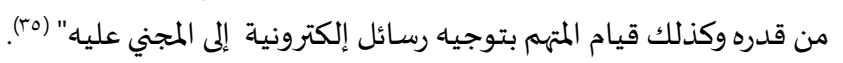

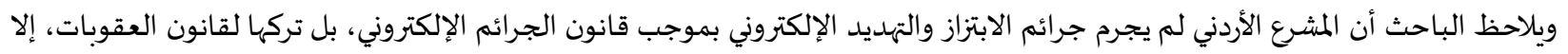

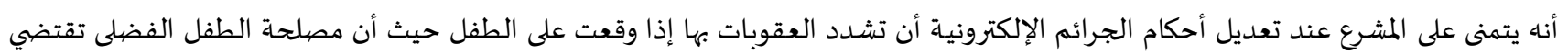

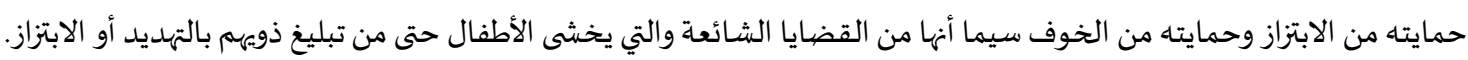

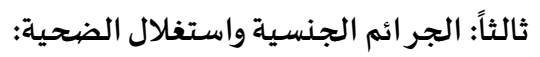

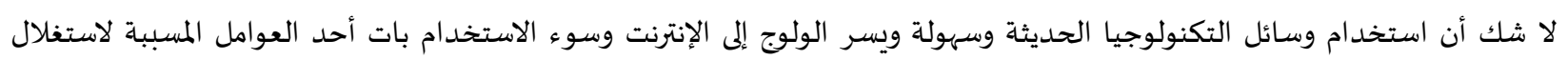

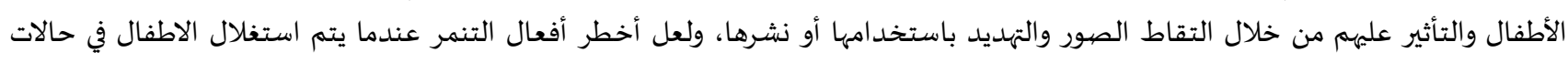

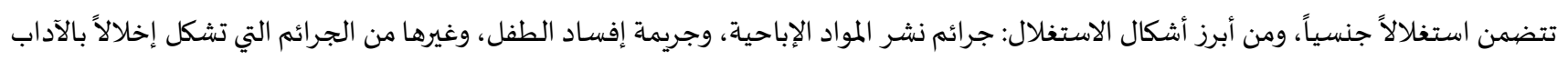

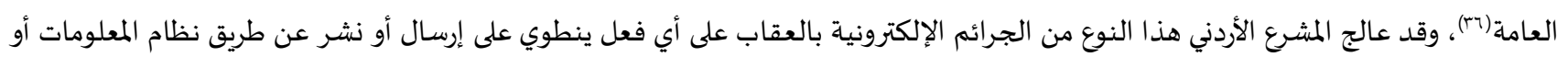

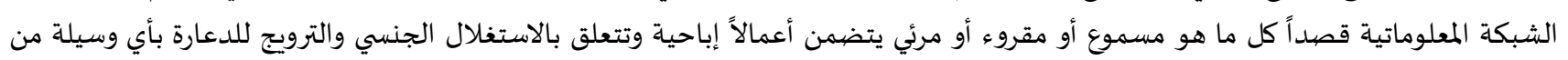

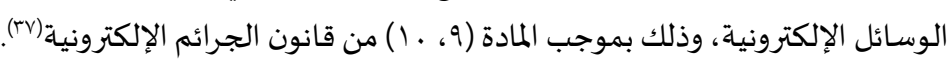

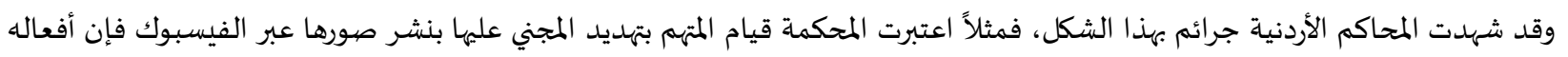

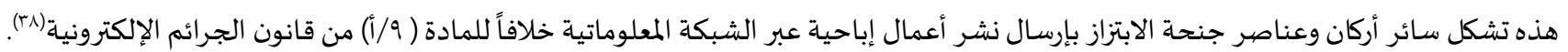

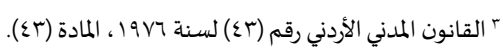

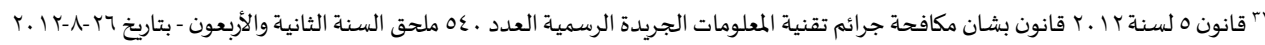

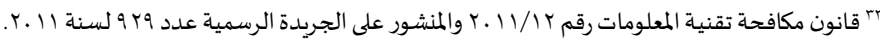

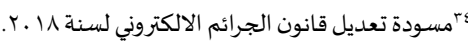

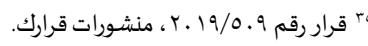

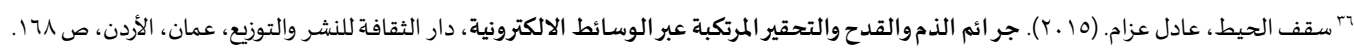

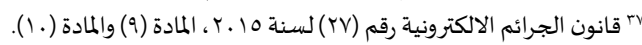

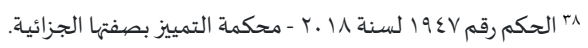




$$
\text { رابعاً: الذم والقدح والتحقير: }
$$

لعل أبرز أفعال المتنمر إلكترونيا تتمثل في الحط من مكاحئ مانه الضحية والتقليل من شأنه بين أقرانه وذلك في سبيل فرض السيطرة عليها وإقصائه

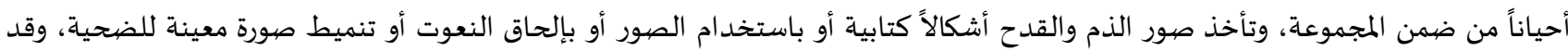

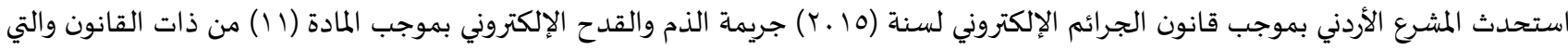

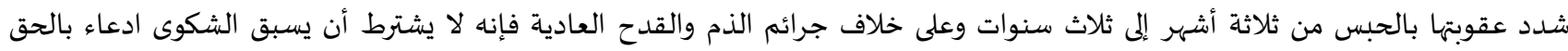

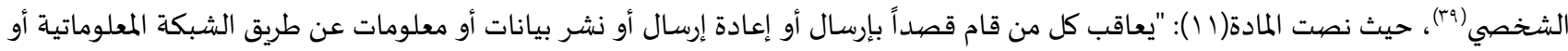
الموقع الإلكتروني أو أي نظام معلومات تنطوي على ذم أو قدح أو تحقير أي شخص بالحبس مدة لا تقل عن ثلاثة أشهر وبغرامة لا تقل عن ( . . ا ( ) مائة دينار ولا تزيد على (.... ( ) ألفي دينار ".

النتائج والتوصيات:

بالنظر لأهمية موضوع الدراسة وحداثته توصلت الدراسة إلى العديد من النتائج والتوصيات كان أبرزها:

ظاهرة التنمر ليست من الظواهر الجديدة فهي قديمة من قدم المجتمعات، وتمارس هذه الظاهرة من قبل فئة أو شخص أكثر قوة على الأشخاص أو الفئات الأقل قوة، وقد تنامت هذه الظاهرة بظهور مواقع التواصل الاجتماعي واستخد ام الوسائل الإلكترونية. يقتضي التنمر القيام بأعمال عدوانية بهدف إلحاق الضرر بالضحية ويظهر من تعريفات التربويون وعلماء النفس أننا إزاء حالة عمدية أو قصدية أي أن نية المتنمر تنصرف إلى إحداث الضرر وبشكل متكرر الحدوث. التنمر الإلكتروني هو إرسال أو نشر نصوص أو صهور ضارة عبر شبكة الإنترنت أو غيرها من الوسائل الرقمية ويتضمن المضايقة ونشر الشائعات وتشويه السمعة أو التنكر والخداع أو الاقصاء. في ظل عدم وجود نص تشريعي يبين ويعرف التنمر الإلكتروني ومن خلال دراسة هذا المدلول والنية به استنتجنا مجموعة من الجرائم التي

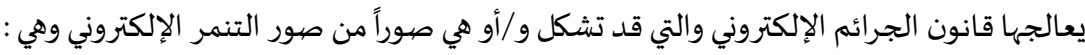

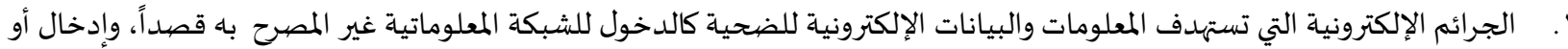

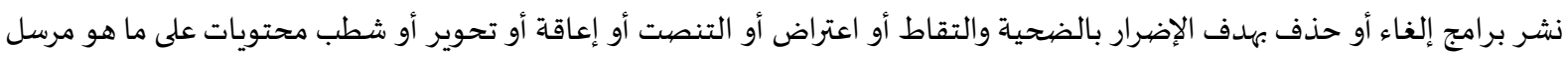
عن طريق الشبكة المعلوماتية أو أي نظام معلومات.

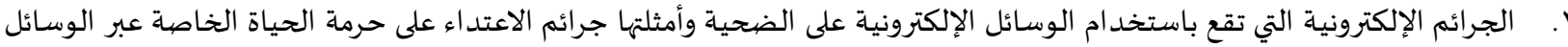

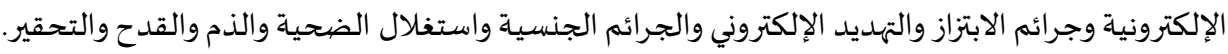
إن قانون الجرائم الإلكتروني تكفي نصهوصه للحدد من هذه الظاهرة إلى حد ما، وعليه فإن قانون الجرائم الإلكتروني يشكل ضمانة لضمانة لضحايا التنمر في مجمل الصور التي تندرج تحت مصطلح التنمر مما يعني كفاية النصوص التجريمية وأن كان لا بد من تغليظ العقوبات لبعض العض الصودي الصور لحماية القصر من التنمر الجنسي.

التوصيات: حيث أن استخدام الانترنت بات يُخرج مصطلحات كالتنمر الإلكتروني مثلاً فإنه يتعين على العاملين بالقانون السعي وراء توضيح هذه

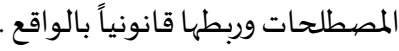
تشديد العقوبات بالجرائم التي ترتكب بحق ضحايا التنمر إذا كانوا أطفالاً، وخاصة جرائم الذم والقدح والتهديد والابتزاز. نتمنى على المشرع الأردني تجريم عقوبة التهديد والابتزاز الواقع من خلال الإنترنت. 


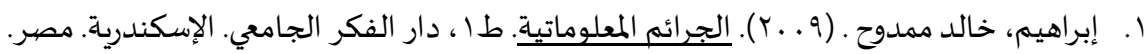

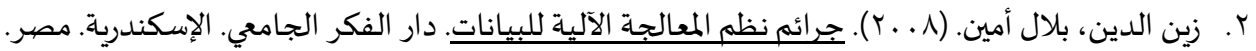

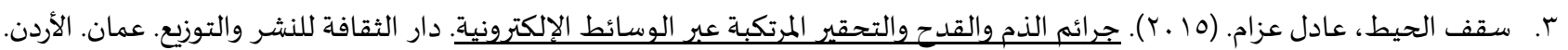

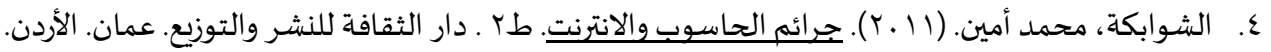

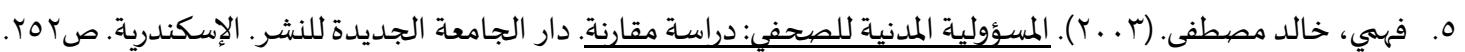
الدراسات والأبحاث

ا. إسلام، عبد الحفيظ محمد عمارة .(Y) . Y). "التنمر التقليدي والإلكتروني بين طلاب التعلم ما قبل الجامعي". مجلة دراسات عربية في التربية

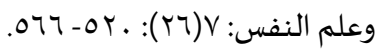

r. الديار، مسعد. (11 .ب). "فاعلية برنامج إرشادي لتقدير الذات في خفض سلوك التنمر لدى الاطفال ذوي اضطراب الانتباه المصحوب بفرط

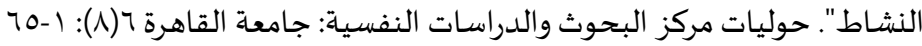

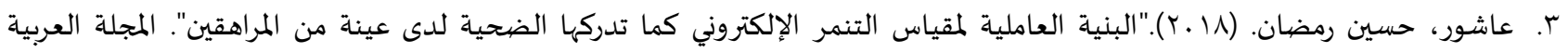

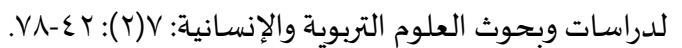

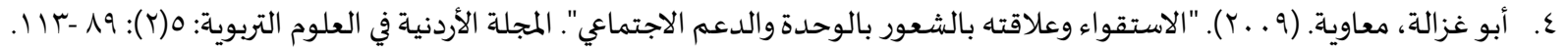

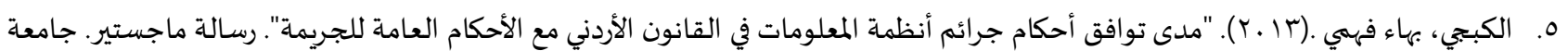

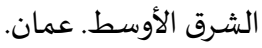

$$
\begin{aligned}
& \text { القو انين والأحكام }
\end{aligned}
$$

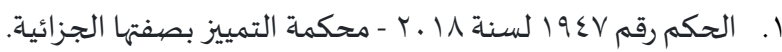

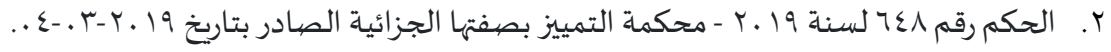

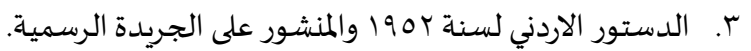

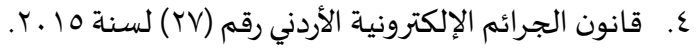

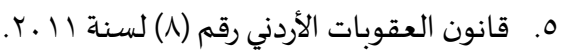

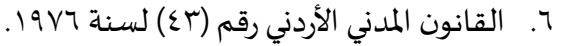

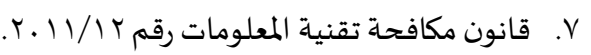

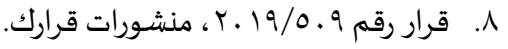

ثانياً : المراجع الأجنبية:

[1] Beran,T \& LI .Q. (2005). "Cyber-Harassment: Study of a new method for an old behavior". Journal of Educational computing Research. 32 (3): 265-277, https://doi.org/10.2190/8yqm-b04h-pg4d-bllh.

[2] Olweus, D. (1993). Bullying at school: what we know and what we can do. Wiley-Blackwell: oxford.

[3] Rosa Mcphee, (2014). "Sticks stones may break my bones, but cyberbullying is illegal - is cyberbullying a crime m and should be". p.4

1. https://www.dosomething.org/us/facts/11-facts-about-cyberbullying?fbclid=IwAR3IE9bJGsGd170ltMjYLj5GOfv]3HgYBvKgPRZosTyzzWSXAyOpMoGiZE, $(22 / 06 / 2020)$

2. https://royanews.tv/news/186142, Date of entry (12/07/2020)

3. Cyberbullying Statistics, Facts, and Trends in 2020, https://firstsiteguide.com/cyberbullying-stats/, Date of entry $(10 / 07 / 2020)$ 


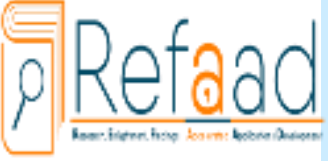

www.refaad.com
المجلة الدولية للدراسات القانونية والفقهية المقارنة

International Journal of Legal and Comparative Jurisprudence Studies (LCJS)

Journal Homepage: https://www.refaad.com/views/LCJS/Home.aspx

ISSN: 2708-6607(Online) 2708-6593(Print)

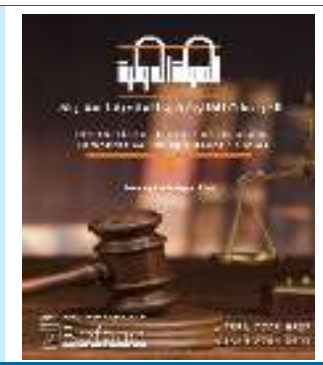

\title{
The sufficiency of electronic legislation to limit electronic bullying- Study in Jordanian legislation
}

\author{
Sakher Ahmad Al-Khasawneh \\ Media Legislations, Jordan Media Institute, Amman, Jordan \\ sakherkhasawneh1234@gmail.com
}

DOI: https://doi.org/DOI:10.31559/LCJS2020.1.2.1

\begin{abstract}
The study reviews the electronic legislation set by the Jordanian legislator that works to reduce cyberbullying, as it dealt with the concept of bullying in general and cyberbullying in particular, and the adequacy of electronic legislation to reduce cyberbullying, identifying the term cyberbullying, and showing the images that constitute cyberbullying that Crimes may constitute according to the Jordanian cybercrime law. The problem of the study emerged by answering the main question that reads: "How adequate is the Jordanian legal legislation to reduce cyberbullying?"

The study was divided into two topics, the first one dealt with the nature of bullying and its images, where it reviewed the concept and images of traditional bullying in the first requirement, and the concept and images of electronic bullying in the second requirement, and the second topic reviewed the legal conditioning of the types and forms of electronic bullying. Which targets the victim's electronic information and data? The second requirement deals with adapting electronic bullying images that target the victim's electronic information and data.

This study used the inductive scientific and analytical method for the provisions of the Jordanian Electronic Crime Law No. 27 of 2015 in order to adapt pictures and shapes that fall under the name of electronic bullying and that may constitute a crime punishable by law.

It was found through this study that most forms and forms of bullying fall under texts punishable by the law of electronic crime, and that the electronic crime law constitutes a guarantee for victims of bullying in the entirety of the images that fall under the term bullying, which means the adequacy of criminal texts, and if the penalties for some images must be increased to protect Minors of sexual bullying.
\end{abstract}

\section{Keywords: cyberbullying; electronic legislation; Jordanian legislation.}

\section{References:}

[1] 'ashwr, Hsyn Rmdan. (2018)."Albnyh Al'amlyh Lmqyas Altnmr Alelktrwny Kma Tdrkha Aldhyh Lda 'ynh Mn Almrahqyn". Almjlh Al'rbyh Ldrasat Wbhwth Al'lwm Altrbwyh Walensanyh: 7(2): 42-78.

[2] Aldyar, Ms'd. (2011). "Fa'lyt Brnamj Ershady Ltqdyr Aldat Fy Khfd Slwk Altnmr Lda Alatfal Dwy Adtrab Alantbah Almshwb Bfrt Alnshat". Hwlyat Mrkz Albhwth Waldrasat Alnfsyh: Jam't Alqahrh 6(8): 1-65.

[3] Ebrahym, Khald Mmdwh. (2009). Aljra'm Alm'lwmatyh. T1, Dar Alfkr Aljam'y. Aleskndryh. Msr.

[4] Eslam, 'bd Alhfyz Mhmd 'marh. (2017). "Altnmr Altqlydy Walelktrwny Byn Tlab Alt'lm Ma Qbl Aljam'y". Mjlt Drasat 'rbyh Fy Altrbyh W'lm Alnfs: 7(26): 520- 566.

[5] Fhmy, Khald Mstfa. (2003). Alms'wlyh Almdnyh Llshfy: Drash Mqarnh. Dar Aljam'h Aljdydh Llnshr. Aleskndryh. S252.

[6] Abw Ghzalh, M'awyh. (2009). "Alastqwa' W'laqth Balsh'wr Balwhdh Wald'm Alajtma'y". Almjlh Alardnyh Fy Al'lwm Altrbwyh: 5(2): $89-113$.

[7] Alkbjy, Bha' Fhmy. (2013). "Mda Twafq Ahkam Jra'em Anzmh Alm'lwmat Fy Alqanwn Alardny M' Alahkam Al'amh Lljrymh". Rsalt Majstyr. Jam't Alshrq Alawst. 'man.

[8] Alshwabkh, Mhmd Amyn. (2011). Jra'm Alhaswb Walantrnt. T2. Dar Althqafh Llnshr Waltwzy'. 'man. Alardn.

[9] Sqf Alhyt, 'adl 'Ezam. (2015). Jra'm Aldm Walqdh Walthqyr Almrtkbh 'br Alwsa't Alelktrwnyh. Dar Althqafh Llnshr Waltwzy'. 'man. Alardn. S 168.

[10] Zyn Aldyn, Blal Amyn. (2008). Jra'm Nzm Alm'aljh Alalyh Llbyanat. Dar Alfkr Aljam'y. Aleskndryh. Msr. 